\title{
BORRELIOSI DI LYME: \\ EVOLUZIONE DELLA DIAGNOSTICA DI LABORATORIO
}

\section{Cinco $M$.}

\section{Laboratorio Spirochete,}

Dipartimento di Scienze Biomediche, Università di Trieste

La diagnosi di malattia di Lyme é fondata principalmente sulla sintomatologia clinica: le indagini microbiologiche tuttavia sono di estremo aiuto per la conferma diagnostica soprattutto in quei casi a sintomatologia sfumata, non tipica. In questi anni un notevole sforzo é stato dedicato alla serologia che rimane l'approccio metodologico più seguito a livello ospedaliero : l'attuale strategia (linee gguida Europee e del CDC) prevede di eseguire una diagnosi a "due steps", ossia una prima analisi dei sieri con tests ELISA e successivamente una conferma dei positivi mediante Western Blot. Con tale sequela si raggiunge una sensibilità dell' $40-90 \%$ ed una specificità del $95 \%$.

L'introduzione dell'antigene espresso da Borrelia in vivo VlsE o di una sua porzione conservata $\mathrm{C} 6$, nella batteria degli antigeni sia in immunoenzimatica che in Western Blot ha portato i livelli di specificità al 100\%. Per quanto riguarda la diagnosi diretta: l'isolamento di Borrelia burgdorferi dal paziente rappresenta il" gol- 
den standard" della diagnostica: esso é tuttavia scarsamente realizzato per una serie di difficoltà tecniche non ultimo il lungo tempo di incubazione della cultura e l'esigenza di un laboratorio attrezzato alla gestione di spirochete vive. Essendo la borreliosi di Lyme un'infezione paucibatterica di maggiore applicabilità risulta essere la PCR. Diversi laboratori ne fanno uso e la metodica non é ancora standardizzata. Abbiamo messo a punto una nested-PCR capace di individuare sequenze di B.burgdorferi in tutti i casi clinici esaminati $(100 \%$ sensibilità $)$ dagli amplificati ottenuti é stata sviluppata una genotipizzazione mediante RFLP, che ha rivelato la presenza di infezioni miste da $B$. afzelii, $B$. garinii e $B$. b. sensu stricto persino nello sesso campione nel 15\% dei pazienti. 